\title{
Expanding the clinical and genetic spectrum of Heimler syndrome
}

\author{
Feng-Juan Gao ${ }^{1,2,3+}$, Fang-Yuan Hu ${ }^{1,2,3+}$, Ping Xu ${ }^{1,2,3}$, Yu-He Qi ${ }^{1,2,3}$, Jian-Kang Li ${ }^{4,5}$, Yong-Jin Zhang ${ }^{1,2,3}$, \\ Fang Chen ${ }^{4,6,7}$, Qing Chang ${ }^{1,2,3}$, Fang Song ${ }^{1}$, Si-Mai Shen ${ }^{8}$, Ge-Zhi Xu ${ }^{1,2,3^{*}}$ and Ji-Hong $\mathrm{Wu}^{1,2,3^{*}}$ (1)
}

\begin{abstract}
Background: Heimler syndrome (HS) is a rare hereditary systemic disorder, partial clinically overlapping with Usher syndrome. So far, our knowledge of HS is very limited, many cases are misdiagnosed or may not even be diagnosed at all. This study aimed to analyze the clinical and genetic characteristics of HS, and to evaluate potential phenotype-genotype correlations.

Results: Two HS cases caused by PEX1 mutations were identified, and a novel likely pathogenic mutation, PEX1 c.895_896insTATA, was found. The main ophthalmic finding of the two patients was consistent with retinitis pigmentosa accompanied by cystoid macular edema, but short axial length and hyperopia were also observed as two previously unreported ocular phenotypes. Analysis of the literature showed that of the 29 HS patients previously reported, 12 had PEX6 mutations, 10 had PEX1 mutations, two had PEX26 mutations, and the remaining patients were not genetically tested. Three novel genotype-phenotype correlations were revealed from analysis of these patients. First, most genotypes of every HS patient include at least one missense variant; second, at least one mutation in PEX1 or PEX6 gene affects the AAA-ATPase region in every HS patient with retinal dystrophy, suggesting AAA-ATPase region is a hypermutable region in patients with a retinal dystrophy; third, there are no significant differences between PEX1-, PEX6-, and PEX26-associated phenotypes.
\end{abstract}

Conclusion: Next-generation sequencing is important for the diagnosis of HS. This study expands the clinical and genetic spectrum of HS, and provides additional insights into genotype-phenotype correlations, which is vital for accurate clinical practice, genetic counseling, and pathogenesis studies.

Keywords: Heimler syndrome, Next-generation sequencing, Genetic diagnosis, PEX1, PEX6, Genotype-phenotype

\section{Background}

Heimler syndrome (HS) was first reported in 1991 by A. Heimler as an inherited syndrome characterized by sensorineural hearing loss (SNHL), enamel hypoplasia, and nail abnormalities [1]. Biallelic mutations in the peroxisomal biogenesis factor 1 gene (PEX1; MIM*602136), peroxisomal biogenesis factor 6 gene (PEX6; MIM*601498), and peroxisomal biogenesis factor 26 gene (PEX26; MIM* 608666) are responsible for HS [2-4]. The proteins they encode function together to control peroxisomal matrix protein import, and mutations of these genes are implicated in peroxisome biogenesis disorders (MIM

\footnotetext{
* Correspondence: drxugezhi@163.com; jihongwu@fudan.edu.cn

${ }^{\dagger}$ Feng-Juan Gao and Fang-Yuan Hu contributed equally to this work.

${ }^{1}$ Eye Institute, Eye and ENT Hospital, College of Medicine, Fudan University,

Shanghai, China

Full list of author information is available at the end of the article
}

phenotypic series PS214100). These are characterized by a deficiency of essential peroxisomal functions or even a complete loss of functional peroxisomes, resulting in a wide range of phenotypes that vary in severity $[5,6]$. Patients with the most serious phenotype present at birth only live a few weeks or months (Zellweger syndrome) [7], while some disorders generally present later in childhood, primarily with leukodystrophy, SNHL, retinal dystrophy, and developmental and cognitive delay. Others may have multiple organ dysfunction and psychomotor impairments including craniofacial dysmorphism, neurological abnormalities, sensory defects, and liver, kidney, and bone abnormalities [5]. Therefore, patients with HS represent the mildest phenotypic subgroup $[8,9]$.

Our current knowledge of HS is very limited, with only 29 reported patients worldwide; 26 of these have genetic sequence information [1-4, 9-14]. HS is also a 
systemic disease with a variety of other co-existing congenital abnormalities, and diagnostic criteria have not been proposed $[2,15]$. The HS clinical phenotype varies, but includes acquired SNHL, amelogenesis imperfecta of the teeth, and retinal dystrophy, partially clinically overlapping with Usher syndrome that is characterized by congenital deafness, retinitis pigmentosa (RP), presence or absence of vestibular dysfunction, but no dental anomalies [4]. Although the number of reported cases is small, this does not reflect a low incidence of disease; rather many cases are misdiagnosed or may not even be diagnosed at all, because of the clinical evaluation alone is especially difficult and often not definitive [4]. Thus, an evidence-based, early, accurate, and fast diagnostic approach is in great need. Fortunately, next-generation sequencing (NGS) methods have been shown to be a powerful tool for the diagnosis of genetic or presumed genetic disorders [16, 17]. In this study, we accurately diagnosed two HS patients using NGS and presented their comprehensive ophthalmic examinations. We further reviewed the varied phenotypes and genotypes of all previously reported cases, and uncovered novel genotype-phenotype correlations.

\section{Methods}

\section{Subjects and ethics statement}

The current study was performed in accordance with the Code of Ethics of the World Medical Association (Declaration of Helsinki) for medical research involving human subjects, and was approved by the Ethics Committee of the Eye \& ENT Hospital of Fudan University. Two HS patients and their relatives were recruited after obtaining informed consent.

\section{Clinical evaluation and sample collection}

Both patients underwent a full ophthalmic examination, including best corrected Snellen visual acuity testing (BCVA), color vision (Ishihara color plate), slit lamp biomicroscopy, tonometry, dilated fundus examination, ultrasound biomicroscopy (UBM), A/B-ultrasound (MD300 L; MEDA Co., Ltd., Tianjin, China), wide-field fundus imaging (Optos PLC, Dunfermline, United Kingdom), spectral domain optical coherence tomography (SD-OCT, Spectralis HRA + OCT, Heidelberg, Engineering Inc., Heidelberg, Germany), full-field electroretinography (ERG, according to the standards of the International Society for Clinical Electrophysiology of Vision; available at www.iscev.org), multifocal ERG (mfERG, LKC Utas E3000 LKC Technologies Inc., USA), and visual field (Humphrey Visual Field Analyzer, Carl Zeiss Inc., CA, USA). Family and medical history including subjective degree of vision loss, age of onset and other related clinical manifestations were recorded.
Blood samples of all the patients and their family members were collected from the peripheral blood and stored at $4^{\circ}$ Cbefore further analysis.

\section{Genetic analyses and confirmation of suspected pathogenic variants}

DNA samples were extracted from whole blood using the FlexiGene DNA Kit (Qiagen, Venlo, The Netherlands) according to the manufacturer's protocol. All participants in this study were subjected to analysis using this panelbased NGS. The capture panel contains exon-capture and untranslated regions (UTRs) of 762 genes that are involved in common inherited eye diseases (BGI-Shenzhen, Additional file 3: Table S1). Acquisition of probe sequences: obtain the exon sequence and its flank $\pm 30 \mathrm{bp}$ of 762 genes from hg38. Each reference sequence begins at one end of a reference sequence and selects the reference sequence of the predetermined length to obtain the probe sequence so that the last total probe can cover the reference sequence at least once, and the probe length of the panel is $90 \mathrm{nt}$, the total target area obtained is $2.3 \mathrm{M}$. On average, the mean coverage depth was more than $400 \mathrm{X}$ and the coverage of target region was around 99.9\% by using BGISEQ-500. Subsequent points for sample quality control have also been added to the probe design process. After sequencing, data analysis was performed as reported previously [18, 19]. Previously reported variants were determined using ClinVar (https://www.ncbi.nlm.nih.gov/clinvar/), the Human Gene Mutation Database (HGMD Professional 2018.4, http://www.hgmd.cf.ac.uk/ac/index.php), and locus-specific databases. Variants were classified as pathogenic and likely pathogenic according to the American College of Medical Genetics (ACMG) and genomics guidelines for the more recent cases. Before confirmation by Sanger sequencing, the candidate variants were reviewed by clinical geneticists and ophthalmologists. Segregation analysis was performed within family members.

\section{Results}

\section{Genetic analyses of the two patients}

Two previously reported mutations, c.2966 T > C (p.Ile989Thr, maternally inherited) and c.2097_2098insT (p.Phe699Phefs*43, paternally inherited) [2,9], were found in patient 1 . A known missense mutation, c.2966 T > C (p.Ile989Thr, maternally inherited) $[2,9]$, and a novel likely pathogenic mutation, c.895_896insTATA (p.Asn299Ilefs"2, paternally inherited), were found in patient 2. Pedigrees and identified mutations of the two families are shown in Fig. 1. We found no additional pathogenic or likely pathogenic variants known to be associated with inherited eye diseases in either patient. 
A

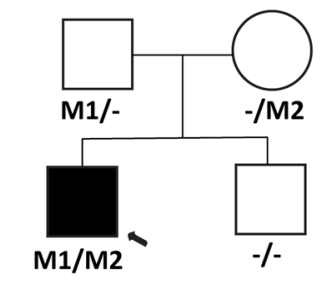

M1: PEX1 c.2966T>C (p.lle989Thr)

M2: PEX1 c.2097_2098insT

(p.Phe699Phefs*43)

Family 1

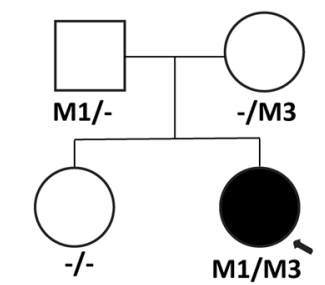

M1: PEX1 c.2966T>C (p.lle989Thr)

M3: PEX1 c.895_896insTATA

(p.Asn299llefs*2)

B
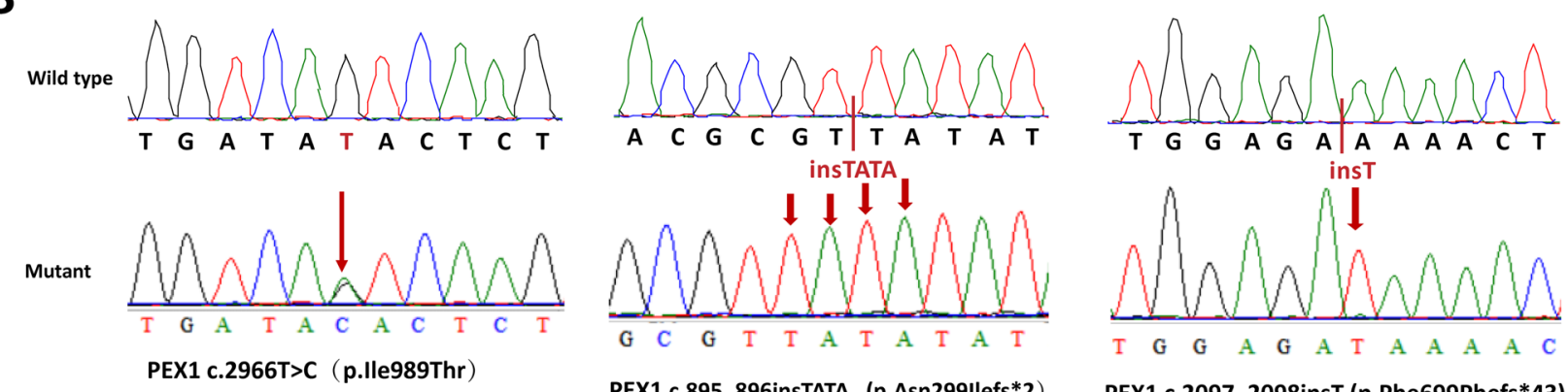

PEX1 c.895_896insTATA (p.Asn299llefs*2)

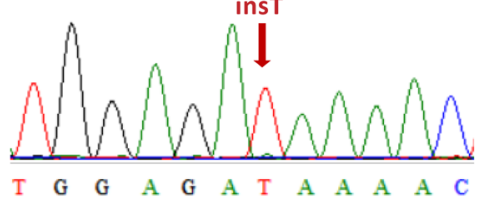

PEX1 c.2097_2098insT (p.Phe699Phefs*43)

Fig. 1 Pedigrees (a) and identified mutations (a). a Circles represent females, and squares represent males. Filled symbols represent affected patients, and empty symbols represent unaffected family members. $\mathbf{b}$ Sequencing results of the mutations in the PEX 1 gene. Arrows indicate the position of the mutated nucleotide

\section{Clinical features of the two patients}

Patient 1 is a 9-year-old boy, and patient 2 is an 8year-old girl. Clinical characteristics of the two patients are summarized in Table 1. Their family history was unremarkable. Both patients had SNHL since birth and amelogenesis imperfecta on their secondary teeth. Nails, development, and intelligence were normal (Additional file 1: Figure S1 and Additional file 2: Figure S2). They suffered from a progressive decrease in visual acuity and nyctalopia since birth. BCVA values of patient 1 and 2 were $0.8 / 0.6$ and $0.4 / 0.5$, respectively, and intraocular pressures were within the normal range. There were no abnormalities in anterior segments. Fundus examinations revealed midperipheral and peripheral retinal pigmentation abnormalities in both patients, consistent with RP (Figs. 2 and 3). SD-OCT showed marked loss and disruption of the ellipsoid zone, photoreceptor outer segment, and retinal pigment epithelium (RPE). Cystoid macular edema (CME) was seen in both patients, although cystoid spaces were larger and more frequent in patient 2 . Interestingly, both patients were previously diagnosed with Usher syndrome as well as attention deficit hyperactivity disorder (ADHD), their axial lengths $(20.69 \mathrm{~mm} / 20.49 \mathrm{~mm}$ vs $20.17 \mathrm{~mm} / 20.30 \mathrm{~mm}$, respectively) were shorter than their peers, and they suffered from hyperopia $(+3.25 /+3.75$ DS vs $+3.75 /+$ 1.65 DS, respectively).

Follow-up visits were scheduled 2 months and 2 years after the first visit of patient 2. At the 2-month visit, CME was aggravated and the central foveal thickness (CFT) increased from 510 to $603 \mu \mathrm{m}$ in the right eye and from 599 to $666 \mu \mathrm{m}$ in the left eye; as a result, the BCVA decreased from $0.6 / 0.7$ to $0.4 / 0.5$. At the third visit 2 years later, the CFT of both eyes had decreased spontaneously; however, through anatomic improvement, there was no notable visual gain (Fig. 4). The thickness of all other retinal layers showed no major change throughout the 2 year-follow-up (Additional file 4: Table S2).

\section{Genotype-phenotype correlations}

Clinical and genetic characteristics of the previously reported HS cases were analyzed and are listed in Table 2. To date, 29 HS patients have been reported worldwide, which gives a total of 31 including the two patients in this study (male $=10$, female $=21$ ). Among the 29 reported HS patients, most derive from the UK $(n=10)$ and the USA $(n=8)$, while only one is of Chinese origin. Twelve patients from seven families harbor PEX6 mutations, 10 patients from eight families carry PEX1 mutations, and two patients from two families have PEX26 mutations. No variants were identified in the currently 
Table 1 Summary of mutations, ophthalmologic findings, and other features of the two patients

\begin{tabular}{|c|c|c|}
\hline & Case 1 & Case 2 \\
\hline Ethnicity/gender & Chinese/male & Chinese/female \\
\hline Age of eye examination (years) & 9 & 8 \\
\hline Mutation & $\begin{array}{l}\text { PEX1 c.2966 T > C; p.lle989Thr } \\
\text { PEX1 c.2097_2098insT; p.Phe699Phefs*43 }\end{array}$ & $\begin{array}{l}\text { PEX1 c.895_896insTATA p.Asn299llefs*2 PEX1 c.2966 T> } \\
\text { C p.lle989Thr }\end{array}$ \\
\hline Family history & Unremarkable & Unremarkable \\
\hline \multicolumn{3}{|l|}{ Ophthalmic findings } \\
\hline$-1 \mathrm{OP}$ (mmhg) & $10 / 10$ & $9 / 10$ \\
\hline -BCVA & $0.8 / 0.6$ & $0.4 / 0.5$ \\
\hline - Cycloplegic refraction & $\begin{array}{l}R:+3.25 /+2.75 \times 85 \\
L:+3.75 /+3.00 \times 85\end{array}$ & $\begin{array}{l}R:+3.75 /-0.5 \times 67 \\
L:+1.65 /-0.37 \times 88\end{array}$ \\
\hline - Color amblyopia & Color amblyopia & N \\
\hline - Cornea & $\mathrm{N}$ & $\mathrm{N}$ \\
\hline -Corneal curvature & $\begin{array}{l}\text { R: } 41.11 / 45.36 \mathrm{D} \\
\text { L: } 41.01 / 44.53 \mathrm{D}\end{array}$ & $\begin{array}{l}\text { R: } 45.55 / 46.30 \mathrm{D} \\
\text { L: } 45.98 / 46.49 \mathrm{D}\end{array}$ \\
\hline -Central corneal thickness & 563 um/563 um & NA \\
\hline - Anterior chamber depth & $2.65 \mathrm{~mm} / 2.54 \mathrm{~mm}$ & $1.99 \mathrm{~mm} / 1.99 \mathrm{~mm}$ \\
\hline - Iris & $\mathrm{N}$ & $\mathrm{N}$ \\
\hline - Axial lengths & $20.69 \mathrm{~mm} / 20.49 \mathrm{~mm}$ & $20.17 \mathrm{~mm} / 20.30 \mathrm{~mm}$ \\
\hline - Lens & $\mathrm{N}$ & $\mathrm{N}$ \\
\hline$-U B M$ & $\mathrm{~N}$ & $\mathrm{~N}$ \\
\hline - Retinal vessels & $\mathrm{N}$ & $\mathrm{N}$ \\
\hline -Retina & $\begin{array}{l}\text { Retinal atrophy in the mid and far periphery } \\
\text { combined with significant bone spicule-like } \\
\text { pigmentation }\end{array}$ & $\begin{array}{l}\text { Retinal atrophy in the mid and far periphery } \\
\text { combined with significant bone spicule-like } \\
\text { pigmentation }\end{array}$ \\
\hline - Optic nerve & $\begin{array}{l}\text { The inferionasal rim of the optic disc is blurred } \\
\text { ( } R \text { and } L \text { ) }\end{array}$ & $\begin{array}{l}\text { The inferionasal rim of the optic disc is blurred } \\
(\mathrm{R} \text { and } \mathrm{L} \text { ) }\end{array}$ \\
\hline -B scan & $\begin{array}{l}\text { Light to moderate turbidity in the front and } \\
\text { middle sections of the vitreous, posterior vitreous } \\
\text { detachment }\end{array}$ & $\begin{array}{l}\text { Light turbidity in the front and middle sections } \\
\text { of the vitreous, posterior vitreous detachment }\end{array}$ \\
\hline -RNFL thickness & $\mathrm{N}$ & $\mathrm{N}$ \\
\hline -ffERG & $\begin{array}{l}\text { Profoundly attenuated rod ERGs, subnormal and } \\
\text { delayed cone ERGs. }\end{array}$ & $\begin{array}{l}\text { Undetectable rod ERG, subnormal bright flash } \\
\text { ERG, subnormal and delayed cone ERG. }\end{array}$ \\
\hline -mfERG & $\begin{array}{l}\text { Amplitudes in posterior-pole of both eyes were } \\
\text { significantly reduced, more seriously in the } \\
\text { right eye }\end{array}$ & $\begin{array}{l}\text { Amplitudes in posterior-pole of both eyes were } \\
\text { significantly reduced, more seriously in the } \\
\text { right eye }\end{array}$ \\
\hline -VEP & Reduced of P100 amplitude. & Prolongation of latency and lowness of amplitude. \\
\hline - Humphrey visual field & Peripheral visual field loss, with small central loss & $\begin{array}{l}\text { Peripheral visual field loss, except for the superior } \\
\text { nasal quadrant }\end{array}$ \\
\hline - Goldman visual field & NA & Peripheral visual field constriction \\
\hline -SD-OCT & $\begin{array}{l}\text { Centrally preserved ISe band with small retinal } \\
\text { cysts of the inner nuclear layer }\end{array}$ & Multiple large intraretinal cysts \\
\hline - Orbital MRI & N & N \\
\hline - Sensorineural hearing loss & + & + \\
\hline
\end{tabular}


Table 1 Summary of mutations, ophthalmologic findings, and other features of the two patients (Continued)

\begin{tabular}{lll}
\hline & Case 1 & Case 2 \\
\hline -Teeth & Amelogenesis imperfecta & Amelogenesis imperfecta \\
- Nail abnormalities & $\mathrm{N}$ & $\mathrm{N}$ \\
- Intellect & $\mathrm{N}$ & $\mathrm{N}$ \\
- Electroencephalography & The frequency of theta rhythm is slightly increased & $\mathrm{NA}$ \\
- Development & $\mathrm{N}$ & $\mathrm{N}$ \\
- Psychomotor & $\mathrm{N}$ & $\mathrm{N}$ \\
\hline
\end{tabular}

Abbreviations. IOP intraocular pressure, $B C V A$ best corrected visual acuity, $R$ right, $L$ left, $S D$-OCT spectral domain optical coherence tomography, UBM ultrasound biomicroscopy, RNFL retinal nerve fiber layer, MRI magnetic resonance, ffERG full-field ERG, mfERG multifocal ERG, VEP visual evoked potential, ISe inner segment ellipsoid, $N$ normal, $N A$ not assessed, + positive finding

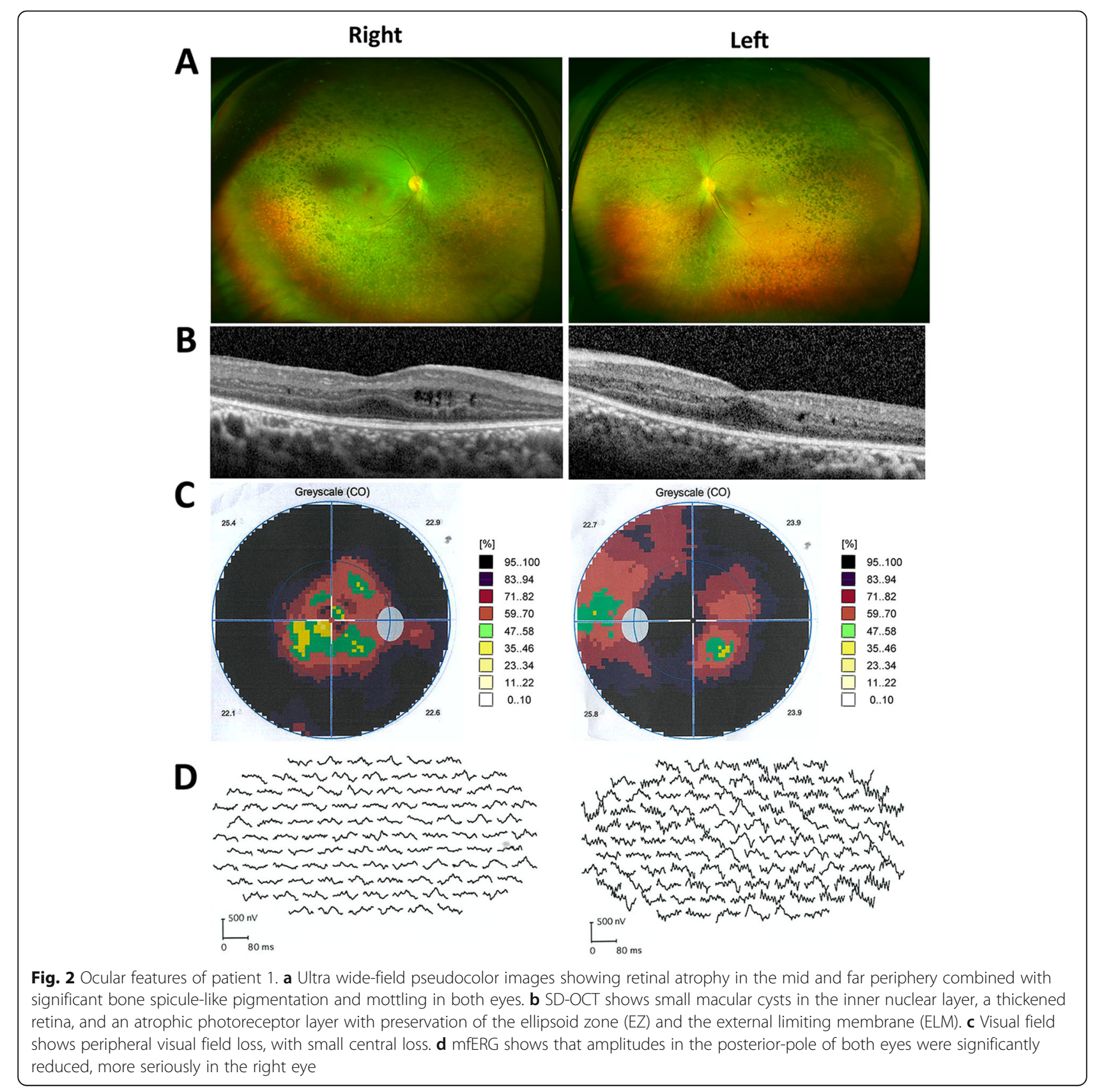




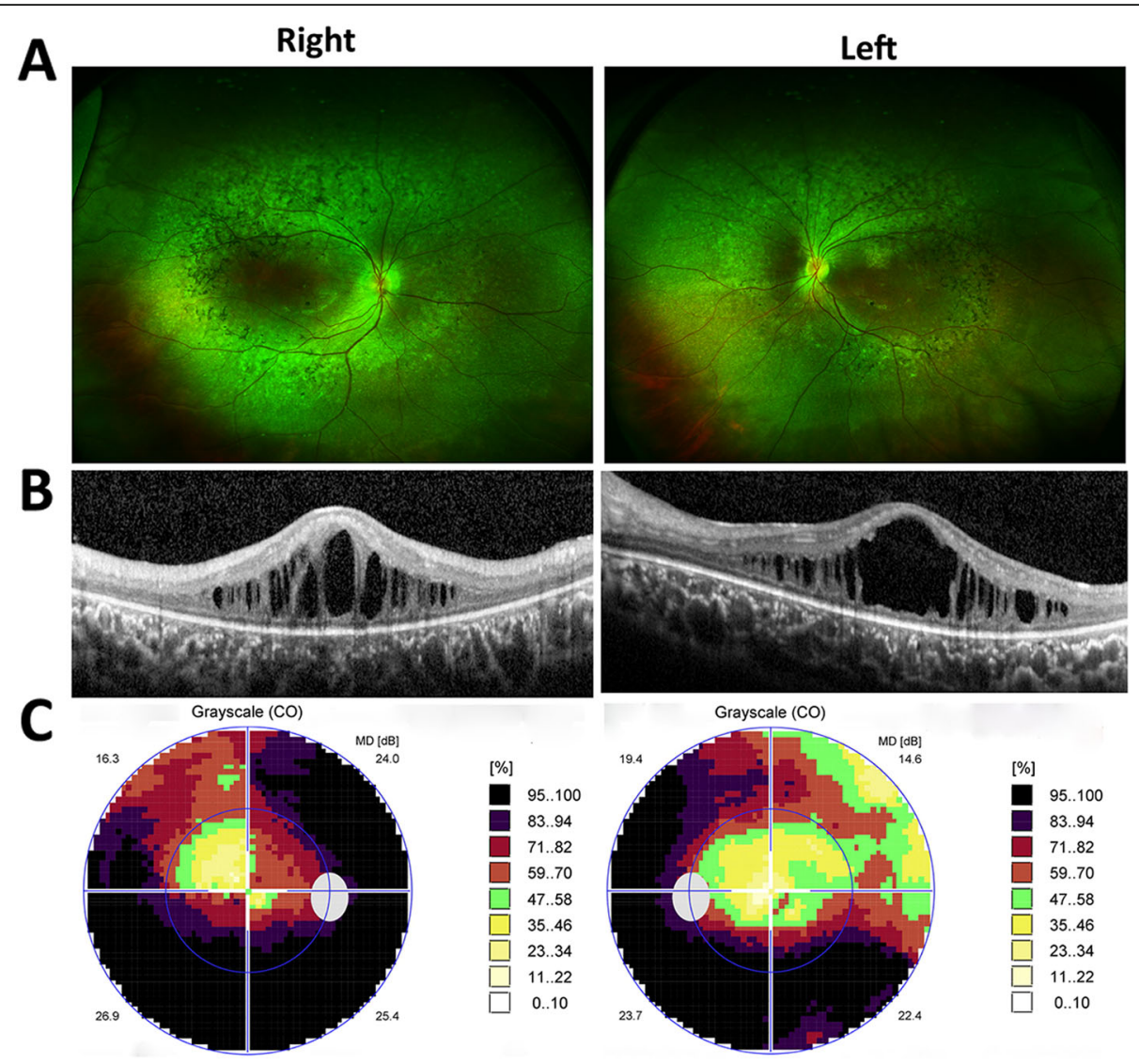

D
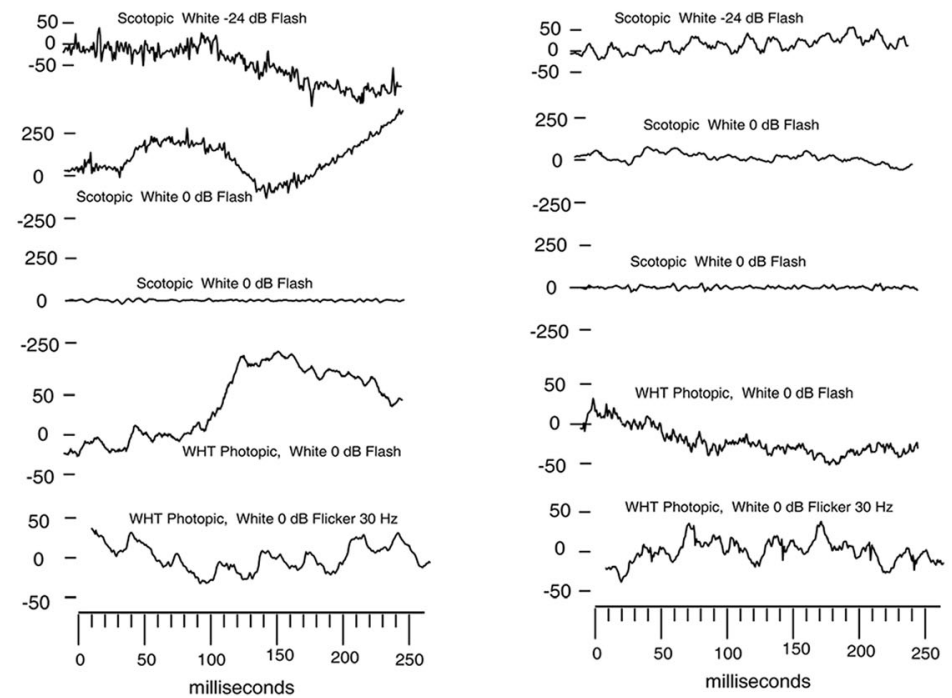

Fig. 3 Ocular features of patient 2. a Ultra wide-field pseudocolor images showing retinal atrophy in the mid and far periphery combined with significant bone spicule-like pigmentation, mottling in both eyes. b SD-OCT shows bilateral cystoid macular edema, which mainly involved the outer nuclear layer (ONL). The foveal and peripheral macular ellipsoid zone (EZ) was disrupted and almost absent. $\mathbf{c}$ Visual field shows peripheral visual field loss except for the superior nasal quadrant. $\mathbf{d}$ mfERG shows undetectable rod ERG, subnormal bright flash ERG, and subnormal and delayed cone ERG

known PEX genes in four patients from two families by exome sequencing [9], while genetic testing was undertaken in three patients from three families. Together with our study, 15 missense mutations $(71.4 \%, P E X 1=4 / 8$, PEX6 $=8 / 10, \quad P E X 26=3 / 3$ ), four frameshift mutations $(19.0 \%, P E X 1=2 / 8, P E X 6=2 / 10)$, one nonsense mutation 


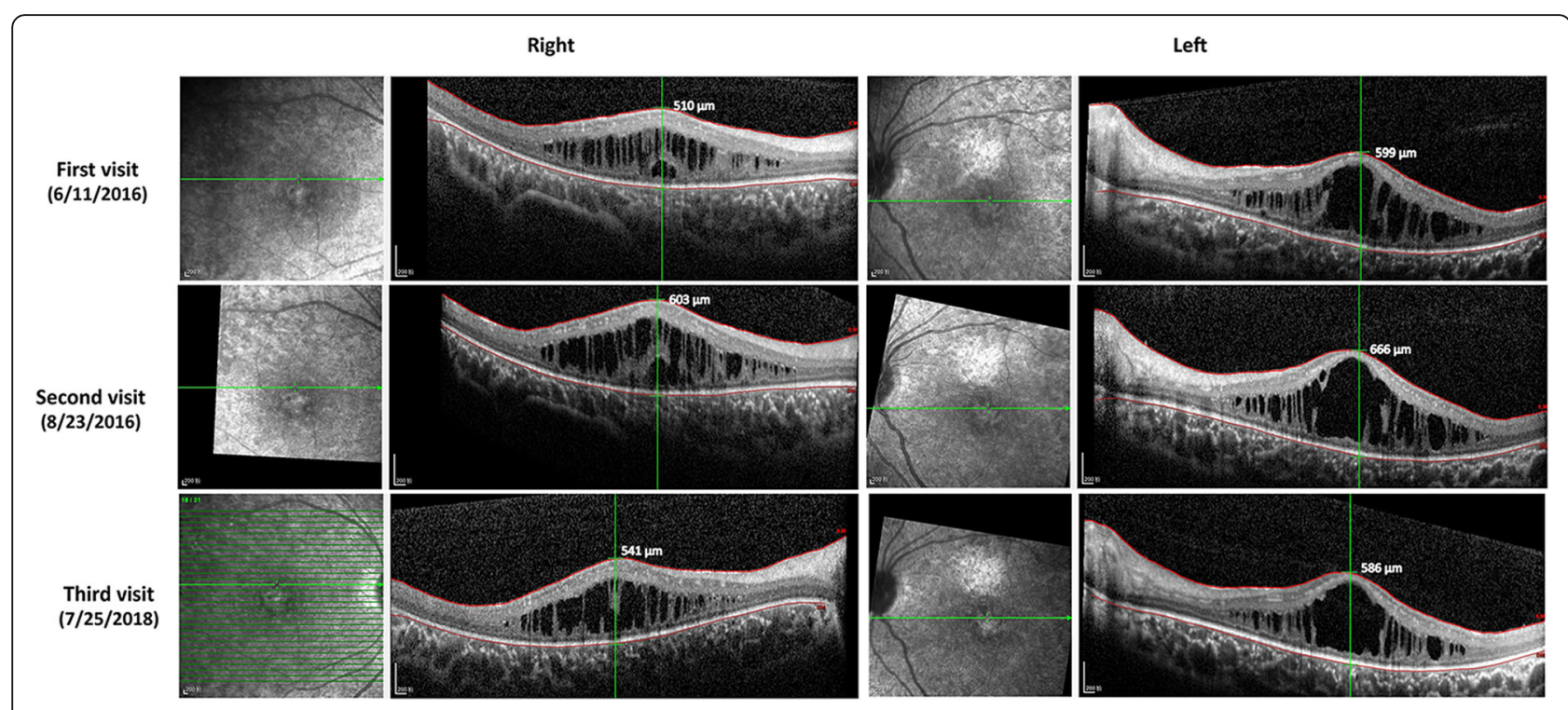

Fig. 4 Follow-up of central foveal thickness in patient 2

(4.8\%, PEX1 $=1 / 8)$, and one splicing mutation (4.8\%, $P E X 1=1 / 8$ ) have been reported to be associated with HS. Only 50 missense mutations (29.4\%, PEX1 $=21 / 85$, PEX6 $=27 / 76, P E X 26=2 / 9)$ were reported to be associated with Zellweger syndrome, which has a severe earlylethal clinical presentation (Additional file 5: Table S3), while the remaining $70.6 \%$ of variants are truncating stop or frameshift defects.

The genotype of every HS patient include at least one missense variant, except for two patients from one family with a homozygous exon 23 nonsense variant, c.3750G > A (p.Trp1250*). This variant is only 19 bases from the last exon-exon boundary of PEX1, and it is likely that the resultant transcript escapes nonsensemediated decay with little effect on protein function, resulting in a mild phenotype. We found a genotypephenotype relationship in HS patients with retinal dystrophy that at least one mutation in PEX1 or PEX6 gene affected the nucleotide sequence of the AAA-ATPase region in every patient, which is typically involved in binding of substrates or cofactors and is vital for the PEX function ${ }^{19-21}$. These results suggesting that AAAATPase region may is a hypermutable region in patients with retinal dystrophy. The locations of these variants are shown in Fig. 5.

All 31 patients were diagnosed with enamel defects of the secondary teeth and SNHL. Twenty patients had RP with or without macular cystoid dystrophy, while no fundus examination was performed on the other four patients so it is unknown if they had RP. Funduscopy of five patients from two families was normal. Twelve patients have nail abnormalities, suggesting that this is not an essential phenotype so should not be a clinical diagnostic indicator for HS [12]. HS was shown to be a rare disease with high phenotypic heterogeneity, with no significant difference between PEX1-, PEX6-, and PEX26-associated phenotypes.

\section{Discussion}

Phenotypic information about individual patients is often insufficiently detailed or inaccessible, thus obstructing the diagnosis of rare systemic diseases or those with overlapping phenotypes. Gene panel sequencing achieves high diagnostic rates in the context of a specific suspected disease or group of diseases, and genetic results can provide support for clinical diagnosis, modify future disease risk, and inform the customization of a variety of therapies $[16,17]$.

So far, our clinical knowledge and understanding of HS are very limited and a diagnosis can easily be overlooked or misdiagnosed. In this work, two patients previously diagnosed with Usher syndrome achieved an accurate diagnosis of HS based on NGS. Additionally, a novel pathogenic mutation and two unreported ocular phenotypes were recognized, and four novel genotypephenotype correlations of HS were uncovered. This provides a deeper understanding of the clinical manifestations and pathogenesis of HS, which is vital for accurate clinical practice, genetic counseling, and pathogenesis studies.

No details of HS ophthalmic manifestations caused by PEX1 mutations have been reported so far. In this work, we present comprehensive ophthalmic examinations of two HS patients induced by PEX1 mutations. As well as the previously reported phenotypic features of RP with CME, these patients also showed short axial lengths and 


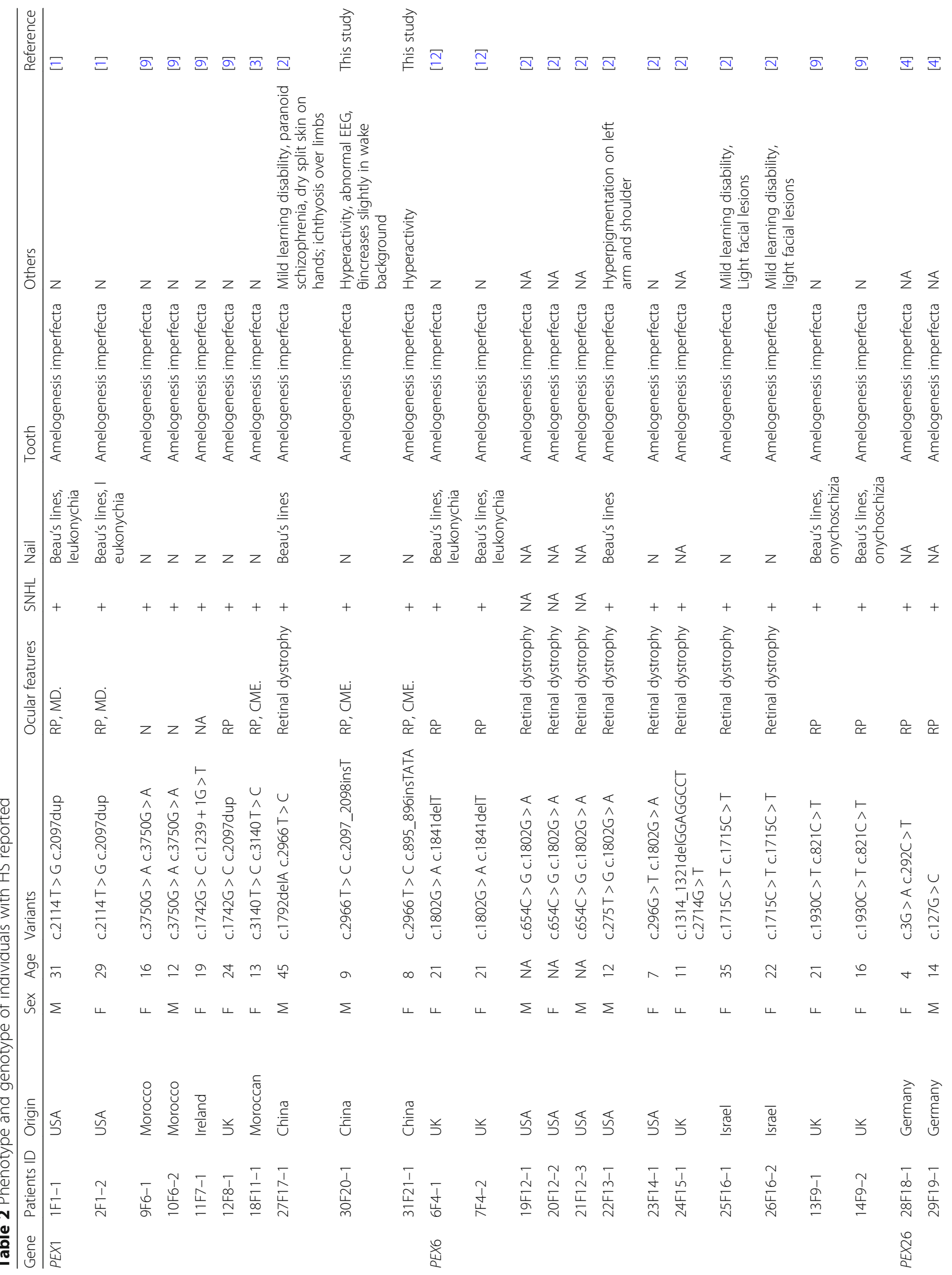




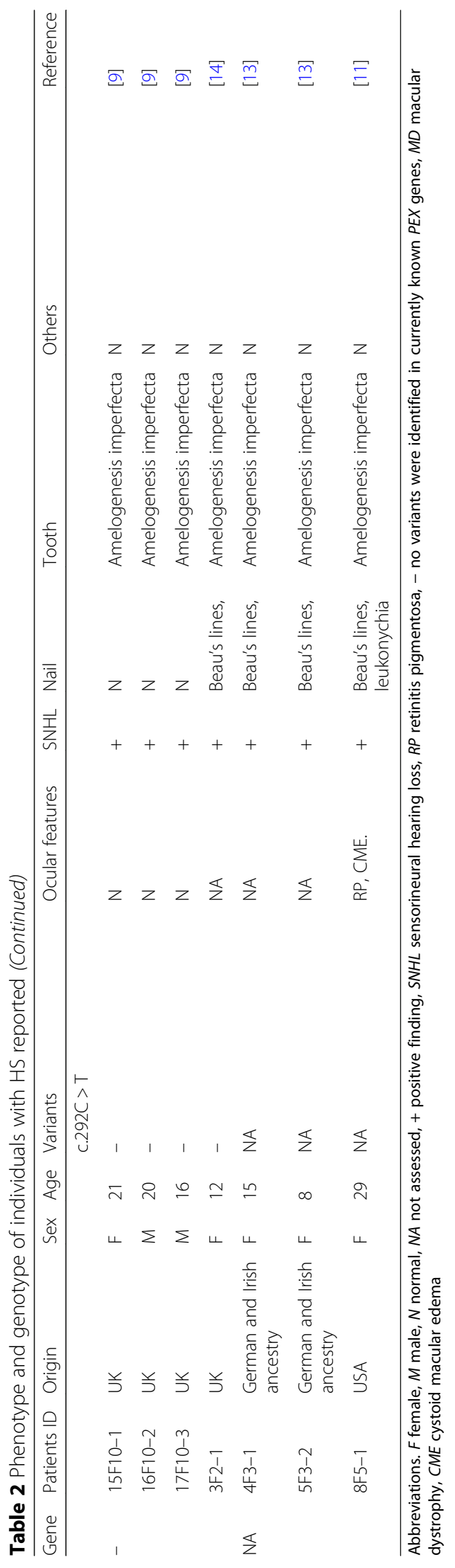




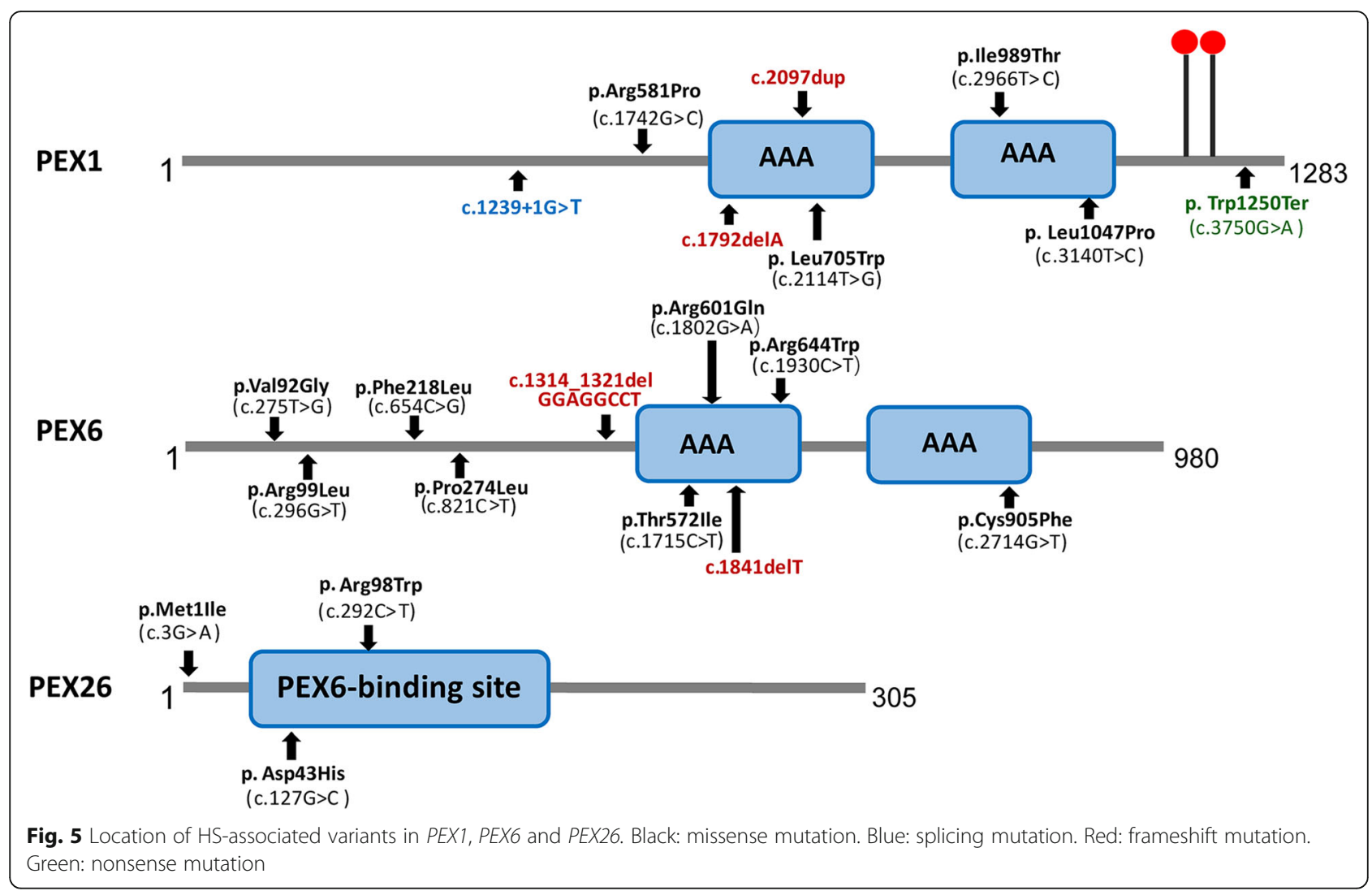

hyperopia suggesting that PEX1 mutation-associated ocular involvement can be developmental (short axial length) and degenerative (RP). Both patients also had ADHD which may be a novel phenotype of HS. After comprehensively analyzing the varied phenotypes of all reported HS cases in the literature, we propose that HS is a systemic disorder with high phenotypic variability; however, all cases demonstrate SNHL (severe to profound) and enamel hypoplasia of secondary dentition. Most patients show infantile-onset retinal dystrophy, and nails are normal or abnormal. They may also suffer from other systemic abnormalities such as learning disabilities or ADHD. Considering the wide clinical heterogeneity, rapid and definitive diagnosis of $\mathrm{HS}$ is challenging, and it is especially difficult to establish clinical diagnostic criteria. NGS can help resolve these challenges, and we suggest that it should become the "gold standard" for diagnosing HS.

Through a comprehensive analysis of the reported mutant genes we found that mutations in PEX1 and PEX6 are the main cause of HS. A small number of cases are also caused by mutations in PEX26, and it is likely that other HS-causing genes remain to be discovered. PEX1 and PEX6 are members of the AAA protein family that are involved in binding ATP to form a heterohexameric ATPase which is associated with various cellular activities that fuel essential protein transport across peroxisomal membranes
$[20,21]$. They also play a key role in matrix protein import from heterohexamers and ternary complexes with PEX26 [22]. Mutant PEX6 and PEX1 proteins result in abnormal peroxisomal function, leading to the accumulation of very long chain fatty acids in photoreceptors, RPE, and pigment-laden macrophages [10, 23].

Genotype-phenotype analysis in this study revealed that HS is caused by genotypes including at least one missense variant, while Zellweger syndrome was caused by more deleterious genotypes, such as truncating stop or frameshift defects. Furthermore, at least one mutation in one patient affects the AAA-ATPase region (PEX1 and PEX6) or PEX6 binding site (PEX26) when HS patients have retinal dystrophy. Together, these results we speculate that hypomorphic mutations in $P E X$ result in a partially functional protein $[24,25]$, mutations in AAA-ATPases play a role in retinal dystrophy, and AAA-ATPase region is a hypermutable region in patients with a retinal dystrophy. No significant difference was found among PEX1-, PEX6-, and PEX26associated phenotypes, perhaps because these genes closely interact in vivo so any mutation in a given gene affects the function of the entire complex [26, 27]. It is also conceivable that a single genetic locus cannot explain the full phenotypic spectrum, and we propose that additional genetic and possibly nongenetic modifiers cause the various phenotypes. 
One limitation of this study is that the genotypephenotype correlations were based on only $31 \mathrm{HS}$ patients. Therefore, these correlations should be confirmed in more HS patients in future studies. Additionally, only one HS patient was followed up over a 2-year period, which may not accurately reflect the progress of the disease. Longer follow-up in more HS patients is required to better understand the ocular prognosis of this disease.

\section{Conclusions}

To summarize, we report two HS families diagnosed by NGS and present the first comprehensive report of the HS ophthalmic phenotype. We identified a novel pathogenic mutation and two unreported ocular phenotypes, which expands the known mutation and clinical spectra of HS. Furthermore, we propose that HS is a systemic disorder with high phenotypic heterogeneity, and show the importance of NGS in the diagnosis of HS. Three novel genotype-phenotype correlations of HS were also uncovered, which is useful for accurate clinical practice, genetic counseling, and pathogenesis studies. These data should be validated and expanded in additional studies.

\section{Supplementary information}

Supplementary information accompanies this paper at https://doi.org/10. 1186/s13023-019-1243-x.

Additional file 1: Figure S1. Clinical characteristics of patient 1. A and $B$ : the hearing test demonstrated the presence of sensorineural hearing loss. C and D: The clinical appearance of teeth and orthopantograms show severe amelogenesis imperfecta. E: Fingernails and toenails are normal, and no Beau's lines or leukonychia were observed.

Additional file 2: Figure S2. Clinical characteristics of patient 2. A and $\mathrm{B}$ : The hearing test shows that both ears suffered from SNHL since birth. C and D: The clinical appearance of teeth and orthopantograms show severe amelogenesis imperfecta. E: Fingernails and toenails are normal, and no Beau's lines or leukonychia were observed.

Additional file 3: Table S1. Gene list of capture panel (762 genes). Additional file 4: Table S2. Follow-up of intraretinal layer thickness in patient 2.

Additional file 5: Table S3. Reported pathogenic variants in PEX1, PEX6, and PEX26 in the Human Gene Mutation Database associated with Zellweger syndrome.

\section{Abbreviations}

ACMG: American College of Medical Genetics; ADHD: Attention deficit hyperactivity disorder; ATP: Adenosine triphosphate; BCVA: Best corrected visual acuity testing; CFT: Central foveal thickness; CME: Cystoid macular edema; ERG: Full-field electroretinography; HGMD: Human Gene Mutation Database; HS: Heimler syndrome; IOP: Intraocular pressure; NGS: Nextgeneration sequencing; OMIM: Online Mendelian Inheritance in Man; PBDs: Peroxisome biogenesis disorders; RP: Retinitis pigmentosa; RPE: Retinal pigment epithelium; SD-OCT: Spectral domain optical coherence tomography; SNHL: Sensorineural hearing loss; UBM: Ultrasound biomicroscopy; UTRs: Untranslated regions

\section{Acknowledgements}

We would like to thank all patients and their family members for their collaboration. We are grateful to the technical staff at Eye and ENT Hospital of Fudan University for their assistance and BGI-Shenzhen for technical support.

\section{Authors' contributions}

JHW and GZX conceived and designed the experiments. FYH, PX and YHQ collected the clinical samples. FJG, JKL and FC analyzed sequencing data. YHQ, YJZ, QC and FJG recruited patients, performed clinical examination of patients and clinical interpretation. FJG, GZX and FYH contributed data for the analysis, discussed and interpreted the results. JHW and FJG drafted and revised the manuscript. All authors read and approved the manuscript.

\section{Funding}

This work was supported by the National Natural Science Foundation of China (Grant NSFC81770944, 81770925, 81790641, the Non-profit Central Research Institute Fund of Chinese Academy of Medical Sciences 2018PT32019).

\section{Availability of data and materials}

Please contact authors for data requests.

\section{Ethics approval and consent to participate}

The study was in accordance with the Declaration of Helsinki and approved by the Ethics Committee of the Eye and ENT Hospital of Fudan University.

\section{Consent for publication}

Written informed consent was obtained from all the subjects or their guardians.

\section{Competing interests}

The authors declare that they have no competing interests.

\section{Author details}

'Eye Institute, Eye and ENT Hospital, College of Medicine, Fudan University, Shanghai, China. ${ }^{2}$ Shanghai Key Laboratory of Visual Impairment and Restoration, Science and Technology Commission of Shanghai Municipality, Shanghai, China. ${ }^{3}$ Key Laboratory of Myopia (Fudan University), Chinese Academy of Medical Sciences, National Health Commission, Shanghai, China. ${ }^{4}$ BGI-Shenzhen, Shenzhen, China. ${ }^{5}$ Department of Computer Science, City University of Hong Kong, 83 Tat Chee Ave, Kowloon, Hong Kong. ${ }^{6}$ Laboratory of Genomics and Molecular Biomedicine, Department of Biology, University of Copenhagen, Copenhagen, Denmark. 'Shenzhen Engineering Laboratory for Birth Defects Screening, BGl-Shenzhen, Shenzhen, China.

${ }^{8}$ Sichuan University, Chengdu, China.

Received: 14 May 2019 Accepted: 29 October 2019 Published online: 12 December 2019

\section{References}

1. Heimler A, Fox JE, Hershey JE, Crespi P. Sensorineural hearing loss, enamel hypoplasia, and nail abnormalities in sibs. Am J Med Genet. 1991;39(2):192-5.

2. Smith CE, Poulter JA, Levin AV, Capasso JE, Price S, Ben-Yosef T, Sharony R, Newman WG, Shore RC, Brookes SJ, et al. Spectrum of PEX1 and PEX6 variants in Heimler syndrome. Eur J Human Genet. 2016;24(11):1565-71.

3. Ratbi I, Jaouad IC, Elorch H, Al-Sheqaih N, Elalloussi M, Lyahyai J, Berraho A, Newman WG, Sefiani A. Severe early onset retinitis pigmentosa in a Moroccan patient with Heimler syndrome due to novel homozygous mutation of PEX1 gene. Eur J Med Genet. 2016;59(10):507-11.

4. Neuhaus C, Eisenberger T, Decker C, Nagl S, Blank C, Pfister M, Kennerknecht I, Muller-Hofstede C, Charbel Issa P, Heller R, et al. Nextgeneration sequencing reveals the mutational landscape of clinically diagnosed usher syndrome: copy number variations, phenocopies, a predominant target for translational read-through, and PEX26 mutated in Heimler syndrome. Mol Genet Genomic Med. 2017;5(5):531-52.

5. Waterham HR, Ebberink MS. Genetics and molecular basis of human peroxisome biogenesis disorders. Biochim Biophys Acta. 2012;1822(9): $1430-41$.

6. Pedrosa AG, Francisco T, Bicho D, Dias AF, Barros-Barbosa A, Hagmann V, Dodt G, Rodrigues TA, Azevedo JE. Peroxisomal monoubiquitinated PEX5 interacts with the AAA ATPases PEX1 and PEX6 and is unfolded during its dislocation into the cytosol. J Biol Chem. 2018;293(29):11553-63.

7. Najafi Sani M, Ahmadi M, Roohani P, Rezaei N. Early onset hepatocellular disease in an infant with Zellweger syndrome. Acta Med Iranica. 2015; 53(10):656-8. 
8. Yik WY, Steinberg SJ, Moser AB, Moser HW, Hacia JG. Identification of novel mutations and sequence variation in the Zellweger syndrome spectrum of peroxisome biogenesis disorders. Hum Mutat. 2009;30(3):E467-80.

9. Ratbi I, Falkenberg KD, Sommen M, Al-Sheqaih N, Guaoua S, Vandeweyer G, Urquhart JE, Chandler KE, Williams SG, Roberts NA, et al. Heimler syndrome is caused by Hypomorphic mutations in the peroxisome-biogenesis genes PEX1 and PEX6. Am J Hum Genet. 2015;97(4):535-45.

10. Wangtiraumnuay N, Alnabi WA, Tsukikawa M, Thau A, Capasso J, Sharony R, Inglehearn CF, Levin AV. Ophthalmic manifestations of Heimler syndrome due to PEX6 mutations. Ophthalmic Genet. 2018;39(3):384-90.

11. Lima LH, Barbazetto IA, Chen R, Yannuzzi LA, Tsang SH, Spaide RF. Macular dystrophy in Heimler syndrome. Ophthalmic Genet. 2011;32(2):97-100.

12. Ong KR, Visram S, McKaig S, Brueton LA. Sensorineural deafness, enamel abnormalities and nail abnormalities: a case report of Heimler syndrome in identical twin girls. Eur J Med Genet. 2006;49(2):187-93.

13. Pollak C, Floy M, Say B. Sensorineural hearing loss and enamel hypoplasia with subtle nail findings: another family with Heimler's syndrome. Clin Dysmorphol. 2003;12(1):55-8.

14. Tischkowitz M, Clenaghan C, Davies S, Hunter L, Potts J, Verhoef S. Amelogenesis imperfecta, sensorineural hearing loss, and Beau's lines: a second case report of Heimler's syndrome. J Med Genet. 1999;36(12):941-3.

15. Ventura MJ, Wheaton D, Xu M, Birch D, Bowne SJ, Sullivan LS, Daiger SP, Whitney $A E$, Jones $R O$, Moser $A B$, et al. Diagnosis of a mild peroxisomal phenotype with next-generation sequencing. Mol Genet Metab Rep. 2016;9:75-8.

16. Splinter K, Adams DR, Bacino CA, Bellen HJ, Bernstein JA, Cheatle-Jarvela AM, Eng CM, Esteves C, Gahl WA, Hamid R, et al. Effect of genetic diagnosis on patients with previously undiagnosed disease. N Engl J Med. 2018; 379(22):2131-9.

17. Adams DR, Eng CM. Next-generation sequencing to diagnose suspected genetic disorders. N Engl J Med. 2018;379(14):1353-62.

18. Kimchi A, Khateb S, Wen R, Guan Z, Obolensky A, Beryozkin A, Kurtzman S, Blumenfeld A, Pras E, Jacobson SG, et al. Nonsyndromic retinitis Pigmentosa in the Ashkenazi Jewish population: genetic and clinical aspects. Ophthalmology. 2018;125(5):725-34.

19. Taylor RL, Parry NRA, Barton SJ, Campbell C, Delaney CM, Ellingford JM, Hall G, Hardcastle C, Morarii J, Nichol EJ, et al. Panel-based clinical genetic testing in 85 children with inherited retinal disease. Ophthalmology. 2017; 124(7):985-91

20. Blok NB, Tan DY, Wang RYR, Penczek PA, Baker D, DiMaio F, Rapoport TA, Walz T. Unique double-ring structure of the peroxisomal PEX1/PEX6 ATPase complex revealed by cryo-electron microscopy. Proc Natl Acad Sci U S A. 2015;112(30):E4017-25

21. Ciniawsky S, Grimm I, Saffian D, Girzalsky W, Erdmann R, Wendler P. Molecular snapshots of the PEX1/6 AAA + complex in action. Nat Commun. 2015;6.

22. Guder P, Lotz-Havla AS, Woidy M, Reiss DD, Danecka MK, Schatz UA, Becker $M$, Ensenauer R, Pagel P, Buttner $L$, et al. Isoform-specific domainorganization determines conformation and function of the peroxisomal biogenesis factor PEX26. Biochim Biophys Acta Mol Cell Res. 2018.

23. Folz SJ, Trobe JD. The peroxisome and the eye. Surv Ophthalmol. 1991;35(5): 353-68.

24. Gootjes J, Schmohl F, Mooijer PA, Dekker C, Mandel H, Topcu M, Huemer M, Von Schutz M, Marquardt T, Smeitink JA, et al. Identification of the molecular defect in patients with peroxisomal mosaicism using a novel method involving culturing of cells at 40 degrees $C$ : implications for other inborn errors of metabolism. Hum Mutat. 2004;24(2):130-9.

25. Imamura A, Shimozawa N, Suzuki Y, Zhang Z, Tsukamoto T, Fujiki Y, Orii T, Osumi T, Kondo N. Restoration of biochemical function of the peroxisome in the temperature-sensitive mild forms of peroxisome biogenesis disorder in humans. Brain Dev. 2000;22(1):8-12.

26. Gardner BM, Castanzo DT, Chowdhury S, Stjepanovic G, Stefely MS, Hurley $\mathrm{JH}$, Lander GC, Martin A. The peroxisomal AAA-ATPase PEX1/PEX6 unfolds substrates by processive threading. Nat Commun. 2018;9(1):135.

27. Gonzalez KL, Ratzel SE, Burks KH, Danan CH, Wages JM, Zolman BK, Bartel B. A PEX1 missense mutation improves peroxisome function in a subset of Arabidopsis PEX6 mutants without restoring PEX5 recycling. Proc Natl Acad Sci U S A. 2018;115(14):E3163-72.

\section{Publisher's Note}

Springer Nature remains neutral with regard to jurisdictional claims in published maps and institutional affiliations.

Ready to submit your research? Choose BMC and benefit from:

- fast, convenient online submission

- thorough peer review by experienced researchers in your field

- rapid publication on acceptance

- support for research data, including large and complex data types

- gold Open Access which fosters wider collaboration and increased citations

- maximum visibility for your research: over $100 \mathrm{M}$ website views per year

At BMC, research is always in progress.

Learn more biomedcentral.com/submissions 